\title{
PERFIL dAS ATITUdes de ALUNOS dO CURSO DE ENFERMAGEM FRENTE A DOENÇA MENTAL, ANTES DA INFLUÊNCIA DA INSTRUÇÃO ACAdêmica, Proveniente de disciplinas de ARea específica
}

\author{
Luiz Jorge Pedrão ${ }^{1}$ \\ Rita de Cássia Avanci \\ Silmara Elaine Malaguti ${ }^{3}$
}

\begin{abstract}
Pedrão LJ, Avanci RC, Malaguti SE. Perfil das atitudes de alunos do curso de enfermagem frente à doença mental, antes da influência da instrução acadêmica, proveniente de disciplinas de área específica. Rev.Latino-am.Enfermagem 2002 novembrodezembro; 10(6):794-9.

Iniciantes no Curso de Enfermagem trazem estereótipos e preconceitos com relação ao portador de doença mental, demonstrando desconhecimento sobre suas possibilidades de recuperação e convívio social. Este estudo objetivou elaborar um perfil de atitudes desses alunos frente a essa pessoa, mostrando aspectos que podem influenciar negativamente nas condutas profissionais futuras. Utilizou-se para isso a escala de opiniões sobre a doença mental. Os alunos exibiram um perfil de atitudes negativo, permitindo concluir que a instrução acadêmica, proveniente de disciplinas dessa área deve ser planejada no sentido de favorecer a mudança do perfil de atitudes apresentado.
\end{abstract}

DESCRITORES: atitudes, enfermagem psiquiátrica, educação em enfermagem

\section{PROFILE OF UNDERGRAdUATE NURSING STUDENTS' ATTITUdES AS TO MENTAL DISEASE BEFORE THE INFLUENCE OF SPECIFIC COURSES}

Students starting the Nursing Course bring with them stereotypes and prejudice in relation to mentally-ill people, thus showing lack of knowledge as to their possibilities of recovery and social living. This study aimed at elaborating a profile of the attitudes presented by these students in relation to such people and at showing aspects that can negatively influence their future professional conduct. To that end, the opinion scale for mental disease was used. The students presented a negative attitude profile, which made it possible to conclude that academic education from courses in this area must be planned so as to favor the change of the attitude profile presented.

DESCRIPTORS: attitudes, psychiatric nursing, nursing education

\section{PERFIL dE LAS ACTITUdES DE ALUMNOS DEL CURSO DE ENFERMERÍA FRENTE A LA ENFERMEDAD MENTAL, ANTES DE LA INFLUENCIA DE LA instrucCIÓN ACAdÉmICA, PROVENIENTE DE DISCIPLINAS de la AREA específica}

Los alumnos que comienzan en el curso de enfermería traen estereotipos y preconceptos con relación al enfermo mental, demostrando desconocimiento sobre sus posibilidades de recuperación y convivencia social. Este estudio buscó elaborar un perfil de actitudes de estos alumnos frente a esta persona, mostrando aspectos que pueden influir negativamente en las conductas profesionales futuras. Los autores utilizaron la escala de opiniones sobre la enfermedad mental. Los alumnos exhibieron un perfil de actitudes negativo, permitiendo concluir que la instrucción académica proveniente de disciplinas de esta área debe de ser planeada en el sentido de favorecer el cambio de perfil actitudinal presentado.

DESCRIPTORES: actitudes, enfermería psiquiátrica, educación en enfermería

\footnotetext{
${ }^{1}$ Professor Doutor, e-mail: lujope@eerp.usp.br; ${ }^{2}$ Alunas de Graduação em Enfermagem. Escola de Enfermagem de Ribeirão Preto da Universidade de São Paulo, Centro Colaborador da OMS para o desenvolvimento da pesquisa em enfermagem
} 


\section{INTRODUÇÃO}

$\boldsymbol{P}$ ara investigar atitude sobre um determinado objeto, doença, ou situação é necessário, a princípio, conceituar esse termo, tendo em vista a não coincidência absoluta sobre a sua definição, por diferentes autores ${ }^{(1)}$.

A atitude do enfermeiro é definida como procedimentos realizados com o propósito de preparar o cliente para os trabalhos clínicos e orientar seu acompanhante, para que possa colaborar na readaptação desse, ao seu meio familiar e social ${ }^{(2-3)}$.

Em relação às atitudes terapêuticas, considerase que essas não são produto de mero instinto, mas sim reações comportamentais, que uma vez formadas por um profissional, deveriam ser incorporadas ao seu modo de ação ${ }^{(4)}$.

Comenta-se que inúmeras definições têm sido propostas para atitude, mas existem várias áreas que exibem uma concordância essencial ${ }^{(5)}$.

Assim, provavelmente, uma grande parte dos autores concordariam que uma atitude pode ser descrita como "predisposição para responder, de maneira consistente favorável ou desfavorável, em relação a um dado objeto", e que a maioria dos instrumentos para medida de atitudes tem base na consistência avaliativa ou afetiva, onde uma pessoa, em diferentes situações, pode exibir diferentes comportamentos em relação a um dado objeto, mas a favorabilidade ou desfavorabilidade, expressa por esses comportamentos, se mantém constante ${ }^{(1)}$.

A definição de atitude, apresentada por esses dois autores, parece ser a mais abrangente dentre as apresentadas por inúmeros outros autores e, dessa forma, mostra-se mais adequada aos propósitos do presente estudo.

O que se entende por doença mental, da mesma forma que para atitude, também tem a necessidade de ser definido, tendo em vista ser um fenômeno cuja maneira de entendimento varia amplamente em diferentes culturas e comunidades, apresentando ainda variações com o passar do tempo ${ }^{(6)}$.

Considerando os questionamentos que surgem quando há a necessidade de definir "dificuldade emocional", "doença mental" ou "distúrbio psiquiátrico" e se diferentes populações compartilham similarmente tais definições, o estudo realizado pela World Federation of Mental Health parece ser um dos mais amplos por tratar-se de investigação transcultural. Esse estudo mostra que a população em geral não distingue os vários tipos de transtornos mentais. Difere apenas o esgotamento nervoso da doença mental, classificando, na primeira categoria, os transtornos originados do meio ambiente e que, submetido a um tratamento de curta duração, teriam "cura". A segunda categoria, mais complexa, afetaria o indivíduo em todos os níveis de desempenho, necessitaria de internações de longa duração, não proporcionando a recuperação completa do paciente, pelo fato de sua personalidade já ter um comprometimento permanente ${ }^{(5)}$.

Pode-se completar essas considerações com aqueles de vários autores, que referem ser o grau de desvio das normas sociais aceitas, prescritas pela sociedade, maior do que a patologia profissionalmente diagnosticada $^{(7)}$. Em outras palavras, a tolerância da sociedade para com o doente mental é desproporcional à real ameaça que a doença mental é para ela.

A população, de forma geral, exibe noções sobre a doença e o doente mental do tipo racionalista (fatores psíquicos ou orgânicos) ou tradicionalista (cérebro fraco), sendo a primeira apresentada por indivíduos que desempenham atividades ocupacionais não manuais e a última, apresentada por indivíduos que apresentam atividades ocupacionais manuais. Estereótipos e preconceitos, como pessoa boba que não raciocina; agressiva, estranha; perigosa e que nunca mais sara; que traz problemas para a família e que tem que ficar no hospício são exibidos também pela população sem correspondência real com os comportamentos que esses doentes emitem no meio social ${ }^{(8)}$.

Alunos ingressantes no Curso de Enfermagem trazem consigo estereótipos e preconceitos semelhantes aos descritos anteriormente, detectado através de suas falas, onde chegam a definir o doente mental como "velho e burro", demonstrando total desconhecimento, tanto com relação à incidência da doença mental nas várias faixas etárias, como em relação à recuperação desse paciente e, ainda, das possibilidades de sua convivência no seu meio social e familiar.

Saber mais sobre as atitudes dos estudantes de enfermagem frente o doente e a doença mental, antes de cursarem qualquer disciplina da Área de Psicologia, Saúde Mental e Enfermagem Psiquiátrica, é um aspecto muito importante, tendo em vista que os programas dessas disciplinas podem ser trabalhados no sentido de oferecer a esses estudantes mais recursos, para que eles tenham melhores condições de ampliar o leque de cuidados a 
serem oferecidos ao paciente em questão. Isso porque estudos realizados ${ }^{(9-10)}$ demonstram que o treinamento, a educação em serviço, cursos e uso de instrumentos de avaliação que estimulem o aumento do conhecimento com relação à doença e ao doente mental influenciam, favoravelmente, na atitude do profissional que assiste esse paciente.

Cabe salientar, ainda, que alguns estudos objetivam comparações transculturais sobre as atitudes de alunos, tanto de medicina, quanto de enfermagem, sobre a doença mental, reafirmando assim a importância dos aspectos citados na formação desses futuros profissionais $^{(1,11)}$

Para o estudo das atitudes desses estudantes frente à doença e ao doente mental e, em particular, os estudantes de enfermagem, a escala de opiniões sobre a doença mental $\mathrm{ODM}^{(12)}$ se mostra adequada, uma vez que suas qualidades psicométricas foram amplamente avaliadas em épocas e culturas diferentes, apresentando bons resultados ${ }^{(13)}$.

Com base na premissa de que a atitude sobre a doença mental é possível de ser modificada, principalmente em estudantes da área da saúde, quando submetidos à instrução acadêmica(1), e em particular na Enfermagem, no momento em que os alunos cursam as disciplinas das áreas citadas anteriormente, e contando ainda com as possibilidades de mudanças atitudinais por parte desses alunos, quando desenvolvem a parte prática de tais disciplinas, devido ao intenso contato que têm com o doente referido e sua respectiva doença e, ainda, a oportunidade que eles têm de observar o professor assistindo a esses pacientes, servindo assim de modelo profissional aos alunos, é que se estabelecem os objetivos deste estudo, descritos a seguir, lembrando ainda que o contato com a doença mental constitui elemento relevante para mudança de atitude frente ao seu portador e associado a outras condições, tais como, conhecimento dessa doença e envolvimento afetivo, por exemplo, produzirão atitudes mais positivas ${ }^{(14)}$.

\section{OBJETIVOS}

Traçar o perfil das atitudes dos alunos do curso de enfermagem frente à doença mental, antes de terem contato com as disciplinas da área de Enfermagem Psiquiátrica e outras afins.
Oferecer subsídios para o planejamento e o desenvolvimento dessas disciplinas, a partir do perfil das atitudes obtido.

\section{METODOLOGIA}

Sujeitos e local

Participaram deste estudo 68 alunos do primeiro semestre do Curso de Enfermagem da Escola de Enfermagem de Ribeirão Preto da Universidade de São Paulo (EERP-USP), no ano de 1998.

Escala de avaliação utilizada

Foi utilizada a escala de atitudes - opiniões sobre a doença mental - ODM ${ }^{(12)}$, traduzida e validada para o nosso meio ${ }^{(5)}$. Essa escala é composta de 51 afirmações do tipo LIKERT e suas opções de respostas variam segundo uma seqüência progressiva de seis pontos de concordância, desde "concordo totalmente" até "discordo totalmente".

Essas afirmações estão agrupadas em sete fatores, que foram denominados ${ }^{(1)}$ da seguinte forma:

A. autoritarismo - reflete a perspectiva de que o doente mental necessita ser isolado de outros pacientes, permanecendo sob portas trancadas e vigilância. Contém tanto o conceito da irrecuperabilidade pessoal e social do doente quanto a idéia de sua periculosidade. Nesse fator estão incluídas nove afirmações;

B. benevolência - traduz a visão de que o doente mental, devido à sua infelicidade, deve ser amparado através de um protecionismo bondoso e paternalista, com base em cuidados, atenção pessoal e conforto material. Nesse fator estão incluídas cinco afirmações;

C. ideologia de higiene mental - representa a idéia de que o doente mental é uma pessoa semelhante às pessoas normais, com diferenças quantitativas, porém não qualitativas. Podem desempenhar atividades especializadas e até cuidar de crianças. Nesse fator estão incluídas onze afirmações;

D. restrição social - traduz a doença mental como uma espécie de defeito hereditário, completamente diferente de outras doenças, cujo portador pode contaminar a família e a sociedade, devendo, portanto, ser protegido através da restrição aos direitos pessoais e sociais do paciente, 
mesmo após a hospitalização. Nesse fator estão incluídas sete afirmações;

E. etiologia interpessoal - explica a doença mental como originária de vivências interpessoais, com maior ênfase para a interação com figuras parentais. Nesse fator estão incluídas seis afirmações;

F. etiologia de esforço mental - reflete a idéia de que a doença mental origina-se do excessivo "esforço cerebral" por meio do trabalho intelectual exagerado, por pensar demais ou por ter pensamentos negativos. Nesse fator estão incluídas oito afirmações;

G. visão minoritária - traduz o conceito de que o doente mental por ser muito diferente das pessoas tidas como normais, pode ser facilmente reconhecido em um agrupamento humano, principalmente pela sua aparência externa. Nesse fator estão incluídas cinco afirmações.

\section{Procedimento}

\section{Aplicação da ODM}

Inicialmente, em sala de aula, todos os alunos matriculados no primeiro semestre do Curso de Graduação em Enfermagem e Obstetrícia da EERP-USP foram convidados, de forma verbal, a responderem à ODM e foi marcada uma data para a realização da aplicação dessa escala em um dia da semana, 40 minutos antes do início da aula de uma disciplina, onde todos os alunos estariam reunidos.

Foi feito contato com o docente responsável pela aula que seguiria a aplicação da escala, no sentido de comunicá-lo sobre a realização da atividade e também pedir sua colaboração, caso houvesse necessidade de tolerância no horário para o início de seu trabalho.

A aplicação da escala aos alunos foi realizada pelos autores do presente estudo, que estiveram também à disposição, nesse momento, para esclarecerem possíveis dúvidas.

Análise dos resultados

Para contagem dos pontos obtidos com a aplicação da ODM, foram utilizadas fórmulas préestabelecidas $^{(15)}$, originando-se, desta forma, os escores fatoriais brutos para cada aluno, nos sete fatores dessa escala. Posteriormente foi realizada uma composição dos escores apresentados pelos alunos, obtendo-se, portanto, os escores fatoriais brutos médios para cada fator.
Por ser difícil trabalhar com os escores, nessa forma, tendo em vista que o número de itens é diferente para cada fator, foi necessária sua transformação para um sistema que permitisse uma apresentação padrão.

Para isso, o sistema Sten (escores Sten) se mostrou adequado pois proporciona uma apresentação padrão dos fatores da escala referida, com variação entre 1 e 10 , média de 5,5 e um desvio padrão igual a $0,5^{(16)}$. As vantagens da utilização desse sistema foram amplamente discutidas $^{(17)}$.

Dessa forma, foi possível a análise dos resultados através dos escores médios, em valores Sten, obtidos pelos sujeitos deste estudo, para cada fator da ODM, permitindo discussão e considerações sobre suas atitudes frente à doença mental.

\section{RESULTADOS}

Os resultados para os sete fatores da ODM apresentados pelos sujeitos do presente estudo (Figura 1) foram os seguintes, em valores Sten: autoritarismo: 6,66; benevolência: 5,86 ; ideologia de higiene mental: 4,69; restrição social: 6,48; etiologia interpessoal: 4,45; etiologia de esforço mental: 6,17; visão minoritária: 5,82.

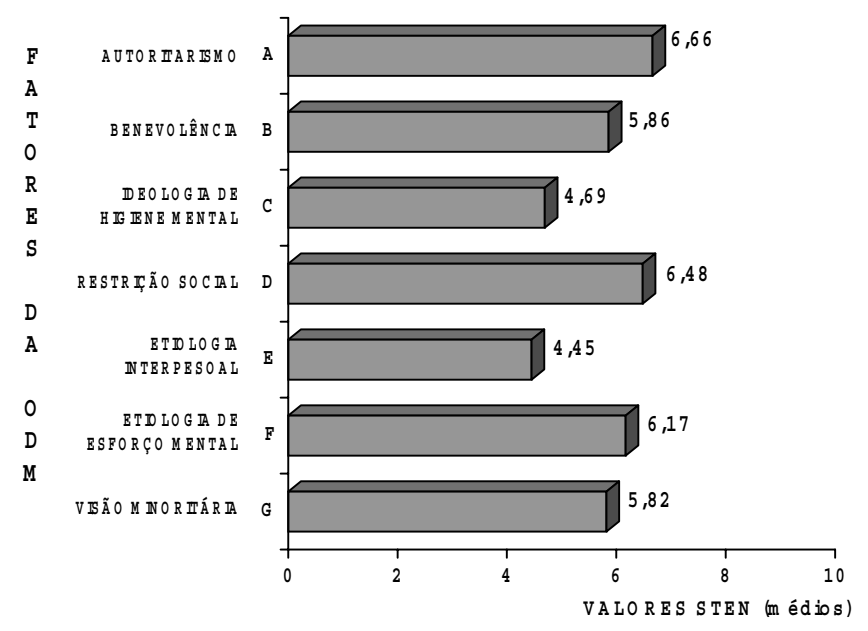

Figura 1 - Perfil de atitudes de alunos do Curso de enfermagem frente à doença mental antes da influência da instrução acadêmica proveniente de disciplinas de área específica

\section{DISCUSSÃO}

A escala de opiniões sobre a doença mental, desenvolvida na década de sessenta ${ }^{(12)}$, mostrou-se 
adequada aos propósitos deste estudo pelo fato de ter sido amplamente utilizada até os dias atuais em diversos trabalhos ${ }^{(1-18-19)}$ e particularmente porque, na década de oitenta, teve a sua tradução e adaptação para o português ${ }^{(5)}$, facilitando o seu uso em nosso meio.

$\mathrm{O}$ estudo das atitudes de alunos iniciantes no curso de enfermagem frente à doença mental e o seu portador é importante pelo fato de não terem ainda influência da instrução acadêmica relativa à formação profissional como um todo e, principalmente, à específica da área de enfermagem psiquiátrica e ciências humanas. Destacar esse aspecto se faz necessário porque os estereótipos e preconceitos em relação à doença e portador citados, trazidos pelos alunos em questão, são basicamente os mesmos evidenciados pela população de forma geral ${ }^{(8)}$, com forte influência de seus meios sócioculturais, em particular o vivenciado pelos alunos estudados, que até há poucos anos esteve dominado por condições políticas extremamente autoritárias e restritivas ${ }^{(1)}$.

Assim, o perfil de atitudes dos alunos sujeitos do estudo no contexto referido mostrou um conteúdo de caráter autoritário, restritivo e discriminador, levando ao entendimento de que, mesmo nos dias atuais, onde se evidencia a real abertura dos grandes hospitais psiquiátricos e que seja extinta a clausura, o portador de doença mental ainda é visto como alguém que apresenta periculosidade, que é irrecuperável e que ainda precisa ser mantido sob portas trancadas e vigilância, ou seja, afastado de seu meio familiar e social.

A tendência à benevolência também é um outro aspecto que compõe o perfil de atitudes dos alunos estudados, revelando, desta forma, não a presença de afetividade ou estima pela pessoa em questão, mas a aderência ao paternalismo caritativo e ao moralismo que estão representados nesse fator.

Os alunos manifestaram desconfiança e descrédito da pessoa portadora de doença mental. Não acreditam também que essa pessoa teve a sua doença desencadeada por vivências interpessoais, principalmente em seu meio familiar. Isso leva ao entendimento de que os alunos tendem fortemente a considerar que o doente mental mostra-se não capacitado para desenvolver atividades, fundamentalmente as mais específicas, que exigem além de técnica, habilidade. Essa tendência é comprovada pela discriminação e rótulo que os alunos impõem ao doente referido, quando se mostram partidários do fato dele ser facilmente reconhecido em um agrupamento humano.

O conjunto das atitudes frente à doença mental, apresentado pelos alunos ingressantes no curso de enfermagem, tem semelhança, de forma geral, com as atitudes apresentadas pelo enfermeiro frente a essa doença, onde há prevalência de conteúdo autoritário, benevolente, restritivo e discriminador, quando comparado a outros profissionais como psicólogos, assistentes sociais e médicos psiquiatras ${ }^{(5)}$. Em um estudo ${ }^{(12)}$ sobre opiniões do pessoal que trabalha em hospital, a respeito da doença mental severa, mostrou que o enfermeiro apresenta médias elevadas do fator benevolência e não figura entre os profissionais que possuem médias satisfatórias de fatores que representam os outros conteúdos citados anteriormente.

Assim, supõe-se que o aluno de enfermagem, mesmo após a influência da instrução acadêmica, conservará certos princípios sobre a doença e o doente mental, que certamente levará para a sua prática profissional, princípios esses que, às vezes, se fazem mais fortes que o treinamento profissional oferecido e determina o desenvolvimento de atitudes frente à doença e o portador referidos $^{(20-22)}$.

Desta forma, o trabalho acadêmico com os alunos deve ser muito cuidadoso no sentido de estar atento aos detalhes descritos, pois sabe-se que as profissões que tendem a assumir com mais facilidade o modelo médico de assistência ao portador de doença mental, e entre elas está a enfermagem, principalmente pela prevalência de seu caráter técnico, possuem conseqüentemente atitudes mais negativas frente a essa pessoa. Em contrapartida, profissionais aderentes ao modelo social de assistência psiquiátrica, entre eles os psicólogos e assistentes sociais, são aqueles que possuem atitudes mais positivas frente à pessoa referida, fato esse, comprovado em alguns estudos $^{(5-23)}$. Cabe salientar que a adoção de um ou outro modelo parece ter ligação com o tipo de treinamento desenvolvido na formação profissional, onde as áreas voltadas às ciências humanas e sociais ressaltam as variáveis sociais de fenômeno e aquelas voltadas ao caráter técnico destacam a volubilidade intrapsíquica ${ }^{(24)}$.

$\mathrm{O}$ conhecimento das atitudes frente à doença mental de alunos de enfermagem, antes de serem influenciados pela instrução acadêmica e principalmente daquela proveniente das áreas específicas citadas anteriormente, se faz necessária porque a modificação de atitudes na situação descrita é possível, principalmente 
em grupos de estudantes da área da saúde ${ }^{(1)}$ e o tipo de treinamento universitário modela as atitudes frente ao doente mental ${ }^{(24)}$.

Vale lembrar que os resultados deste estudo revelam um perfil de atitudes que aponta para o lado negativo, o que na prática profissional poderia ser traduzido em condutas não terapêuticas frente ao portador de doença mental, não contribuindo de modo favorável para a prevenção ou controle dessa doença. É fato, também, que apenas o conteúdo teórico dos cursos não contribui significativamente na positividade das atitudes de alunos frente à doença mental, sendo relevante as vivências práticas e as atitudes de professores no contexto descrito $^{(1-24)}$.

Pode-se concluir que cursos de enfermagem que permitem uma relação professor/aluno mais próxima oferecerão mais oportunidades ao aluno de confrontar suas

\section{REFERÊNCIAS BIBLIOGRÁFICAS}

1. Rodrigues CRC. Comparacion de actitudes de estudiantes de medicina brasileños y españoles hacia la enfermedad mental. Actas Luso-Esp Neurol Psiquiatr y Cienc Afines 1992; 20(1):30-41.

2. Simões N. Da metalinguagem profissional à elaboração de um vocabulário técnico científico na área de enfermagem. [tese]. Ribeirão Preto(SP): Escola de Enfermagem de Ribeirão Preto/USP; 1989.

3. Rodrigues RM. Enfermagem compreendidos como vocação e sua reação com as atitudes dos enfermeiros frente às condições de trabalho. Rev. Latino.am enfermagem 2001; 9(6):76-82.

4. Daniel LF. Atitudes interpessoais em enfermagem. São Paulo (SP): EPU; 1983.

5. Rodrigues CRC. Atitudes frente a doença mental: estudo transversal de uma amostra de profissionais da saúde. [tese]. Ribeirão Preto (SP): Faculdade de Medicina de Ribeirão Preto/ USP;1983.

6. Nunnaly Júnior JC. Popular concepts of mental health, their development and change. New York: Holt, Rinehart \& Winston; 1961.

7. Hood Júnior RW. Dogmatism and opinions about mental illness. Psychol Rep 1973; 32:1283-1290.

8. Alessi NP, Silva GB, Ferreira Santos CA. O doente mental visto pela população de um município paulista. Neurobiologia 1978; 31(3):387-400.

9. Distefano Júnior MK. Pryer MW. Effect of brief training on mental health knowledge and attitudes of nurses and nurses' aides in a general hospital. Nurs Res 1975; 23(1):40-2.

10. Pedrão LJ. Avaliação da escala de observação interativa para pacientes psiquiátricos internados aplicada rotineiramente em uma unidade de psiquiatria de hospital geral. [tese]. Ribeirão Preto (SP): Faculdade de Medicina de Ribeirão Preto/USP; 1997.

11. Smith E. Attitudes of student psychiatric nurses towards mental illness. Nurs Times 1977; 73(30):1174-75. atitudes quando vivenciar as situações descritas anteriormente e, conseqüentemente, mais chances de modificá-las, pois o professor, nesses casos, servirá mais facilmente de modelo de identificação profissional.

Dessa forma, o modo como as disciplinas da área de enfermagem psiquiátrica e outras relacionadas são estruturadas e os seus docentes desenvolvem suas estratégias didáticas, são fatores que podem influenciar nas atitudes dos alunos em questão, cujo perfil apresentado certamente muito contribuirá para a elaboração dos projetos dessas disciplinas. Esses aspectos se fazem importantes no sentido de que esses alunos, no futuro, desenvolvam condutas mais terapêuticas com a pessoa portadora de doença mental, pois é sabido que as atitudes positivas de profissionais na assistência a essas pessoas muito contribui na prevenção da doença citada como também no seu controle.

12. Cohen J, Struening EL. Opinions about mental illness in the personnel of two large mental hospitals. J Abnorm Soc Psychol 1962; 64(5):349-60.

13. Rabkin JG. Opinions about mental illness: a review of literature. Psychol Bull 1972; 77(3):153-71.

14. Pasquali L, Nogueira ALMP, Martins AEO, Martins FMMC. Atitudes frente a doença mental - um modelo etiológico e medida de atitudes (parte 1). Rev Psicol 1987; 5(1):37-67.

15. Struening EL, Cohen J. Fatorial invariance and other psychometric characteristics of five opinions about mental illness fators. Educ Psychol Meas 1963; 23(3):289-98.

16. Canfield AA. The sten scale: a modified C - scale. Educ Psychol Meas 1951; 11:295-7.

17. Lyman HB. Derived Scores. In: Lyman HB. Test scores and what they mean. New Jersey: Prentice - Hall; 1963. p.90136.

18. Kahan AM. Relationship between nurse's opinions about mental illness and experience. Nurs Res 1976; 25(2):13640.

19. Madianos MG, Economou M, Hatjiandreou M, Papageorgion A, Rogakou E. Changes in public attitudes towards mental illnes in the Athens area (1979/1980 - 1994). Acta Psychiatr Scand 1999; 99(1):73-8.

20. Townsend JM. Cultural conceptions, mental disorders and social roles: A comparison of Germany and America. Am Sociol Rev 1975; 40(6):739-52.

21. Koutrelakos J, Gedeon SM, Struening EL. Opinions about mental illness: a comparison of american and greek professionais and laymen. Psychol Rep 1978; 43(3):915-23. 22. Laosebikan S. Atitudes of nigerian and american (U.S.) psychiatric professionals towards the mentally ill. J Soc Psychol 1980; 110(2):291-2.

23. Cooper DG. Psiquiatria e antipsiquiatria. São Paulo: Perspectiva; 1973.

24. Martins AEO. Atitudes frente ao doente mental: influências do tipo e do nível de treinamento universitário. Psicologia: Teoria e Pesquisa 1987; 3(2):92-103. 